\title{
Surgical adverse outcomes and patients' evaluation of quality of care: inherent risk or reduced quality of care?
}

\author{
Perla J Marang-van de Mheen, Nanny van Duijn-Bakker, Job Kievit
}

Qual Saf Health Care 2007;16:428-433. doi: 10.1136/qshc.2006.021071

See end of article for authors' affiliations

\section{Correspondence to} Dr Perla J Marangvande Mheen, Department of Medical Decision Making, Leiden University Medical Center, J10-S, PO Box 9600, 2300 RC Leiden, The Netherlands; p.j.marang@ lumc.nl

Accepted 30 April 2007

\begin{abstract}
Background: Previous research has shown that sicker patients are less satisfied with their healthcare, but specific effects of adverse health outcomes have not been investigated. The present study aimed to assess whether patients who experience adverse outcomes, in hospital or after discharge, differ in their evaluation of quality of care compared with patients without adverse outcomes.

Method: Inhospital adverse outcomes were prospectively recorded by surgeons and surgical residents as part of routine care. Four weeks after discharge, patients were interviewed by telephone about the occurrence of post-discharge adverse outcomes, and their overall evaluation of quality of hospital care and specific suggestions for improvements in the healthcare provided.

Results: Of 2145 surgical patients admitted to the Leiden University Medical Center in 2003, 1876 (88\%) agreed to be interviewed. Overall evaluation was less favourable by patients who experienced postdischarge adverse outcomes only (average 19\% lower). These patients were also more often dissatisfied (OR $2.02,95 \% \mathrm{Cl} 1.24$ to 3.31 ) than patients without adverse outcomes, and they more often suggested that improvements were needed in medical care (OR 2.07, 1.45 to 2.95) and that patients were discharged too early (OR $3.26,1.72$ to 6.20$)$. The effect of inhospital adverse outcomes alone was not statistically significant. Patients with both inhospital and post-discharge adverse outcomes also found the quality of care to be lower (on average $33 \%$ lower) than patients without adverse outcomes.

Conclusions: Post-discharge adverse outcomes negatively influence patients' overall evaluation of quality of care and are perceived as being discharged too early, suggesting that patients need better information at discharge.
\end{abstract}

A dverse outcomes are relevant to both doctors and patients. They are relevant to doctors because adverse outcomes may decrease the quality of care and to patients because they may increase the burden of disease. Patient satisfaction is increasingly considered to be an important indicator of quality of care, ${ }^{12}$ and it is known to be influenced by patient characteristics such as age and health status. ${ }^{3}$ Older patients are usually more satisfied with their hospital stay, but sicker patients report lesser satisfaction. However, evidence about the effect of gender, ethnicity and socioeconomic status is equivocal. ${ }^{3}$ Although health status and health outcomes in general are known to affect patient satisfaction, the specific influence of adverse health outcomes on patient satisfaction has not been investigated to our knowledge. Patients with adverse outcomes may be less satisfied, due to the increased burden of disease or because the possibility of their occurrence had not been communicated. On the other hand, these patients may receive more personal care and attention, and may be more satisfied as a result. The present study aimed to assess:

(1) whether patients who experience adverse outcomes perceive the quality of care differently from patients without adverse outcomes

(2) whether the above relates to adverse outcomes during hospitalisation, after discharge or both.

\section{METHODS}

\section{Study participants and definitions}

We approached all of the 2145 surgical patients admitted to the Leiden University Medical Center in 2003 to participate in the present study. Inhospital adverse outcomes were prospectively recorded throughout admission by surgeons and surgical residents as part of routine medical care, in the standard format of, and following the definition set by, the Dutch nationwide routine reporting programme. ${ }^{45}$ The definition of an adverse outcome as used in this programme is:

\begin{abstract}
"an unintended and unwanted event or state occurring during or following medical care, that is so harmful to a patient's health that (adjustment of) treatment is required or that permanent damage results. The adverse outcome may be noted during hospitalisation, until 30 days after discharge or transferral to another department. The intended result of treatment, the likelihood of the adverse outcome occurring, and the presence or absence of a medical error causing it, are irrelevant in identifying an adverse outcome."
\end{abstract}

This definition is more sensitive than the definition used in most other published studies, ${ }^{6-9}$ as events linked to the natural history of the disease or comorbidity are also included. Its advantage is that it is less prone to discussions on whether this could be expected given the medical condition of the patient, and therefore less likely to result in interobserver variability.

All inhospital adverse outcomes are reported on special forms in patients' records. The presence and completeness of each patient form is checked weekly. Documented information on adverse outcomes is checked and discussed 2 weeks after discharge, in a weekly meeting attended by all surgeons, residents, interns and medical students. The purpose of this discussion is:

- to assess whether the documented information is clear and correct 
- to analyse, interpret and conclude whether anything can be learned from this particular case.

After consensus has been reached, the adverse outcomes are entered into dedicated software and classified by nature, location and possible determinants.

A previous study has shown that inhospital adverse outcomes are effectively identified using this nationwide routine reporting system. ${ }^{4}$ However, given the above definition, adverse outcomes occurring after discharge should also be included but may be missed if these are not reported by patients at their follow-up visit to the outpatient clinic. We therefore interviewed the patients 4 weeks after discharge, including questions on the possible occurrence of adverse outcomes after discharge. The timing of the interview was chosen based on the length of the post-discharge period stated in the definition above. In addition, this length of time was considered to be long enough for any post-discharge adverse outcomes to occur and short enough for patients to recall the adverse outcome clearly.

\section{The interview}

We gave patients information about the study and its purpose during their stay in hospital. Four weeks after discharge, they were contacted by a research assistant for a telephone interview. The interview followed a structured format, and included questions about the occurrence of post-discharge adverse outcomes, the patient's overall evaluation of the quality of care and their specific suggestions for improvements in medical care.

We assessed adverse outcomes in the period up to 30 days after discharge and explained to the patients what was meant by an adverse outcome at the start of the interview. If a patient reported one or more adverse outcomes, we asked them to specify the type of adverse outcome (bleeding, infection, etc.) and checked whether and how it had been treated by a doctor and if it had required readmission or reoperation. In addition, patients were asked to evaluate the overall quality of care they received during their stay in hospital using a scale with grades from 0 to 10 . This grading system is the same as the Dutch educational report card grading system, used throughout the school years and in further education in the Netherlands. We considered a grade below 6 to indicate dissatisfaction, since this cut-off point is always used in the Dutch educational system to decide whether a student has failed an examination or assignment. Since we referred to the educational grading system during the interview, the patients probably would have automatically given a grade according to this system. In other words, if patients graded their hospital stay as 2, they implied that it was not good enough and thus that they were dissatisfied.

Lastly, the interviewer asked the patients an open-ended question regarding specific suggestions on aspects of care most in need for improvement. Patients had to either specify one aspect of care most in need for improvement or could answer that all aspects-surgical and nursing care-were good and did not need to be improved. These suggestions were retrospectively classified into categories (see appendix A for items within categories) by two independent reviewers with good initial agreement $(\kappa=0.83)$. Any differences were discussed until consensus was reached. In line with other questionnaires, ${ }^{10}{ }^{11}$ we discerned the following categories of improvement:

- contact between patient and caregiver, including respectful treatment;

- transfer of information (regarding treatment);

- management issues (eg, waiting times);

- medical care;

- other suggestions.

\section{Statistical analysis}

To answer our first question, we distinguished between patients with and without adverse outcomes (yes/no), regardless of whether the adverse outcome occurred in hospital and/or after discharge. These two groups were compared with respect to overall evaluation, dissatisfaction and suggestions for improvement in the care provided. We then carried out multiple regression analysis to assess whether the differences in the evaluation of care between patients with and without adverse outcomes could be explained on the basis of age, gender and American Society of Anaesthesiologists (ASA) class $^{12}$ of the patient at the first operation during the admission (as a measure of health status of the patient at admission), since these are known to influence patient satisfaction and likely to differ between patients with and without adverse outcomes. Linear regression was used to examine the effect on the overall evaluation, and logistic regression to assess the effect on dissatisfaction (yes/no) and suggesting care improvement (yes/no). Continuous variables (such as age) were first examined to check whether there was a linear relation between the potential confounder and the outcome. Variables showing a non-linear relation with the outcome were categorised. For ASA class, patients were categorised into four groups: not operated, ASA class 1-2, ASA class 3-5 and ASA class missing. Patients with ASA class 3-5 at the first operation were considered as high-risk patients.

We then distinguished between patients who experienced adverse outcomes while in hospital and those who reported adverse outcomes after discharge to assess whether either of these or both influenced the overall evaluation, dissatisfaction and suggestions for improvement. The patients were divided into four groups: patients with inhospital adverse outcomes only; patients with post-discharge adverse outcomes only; patients with both inhospital and post-discharge adverse outcomes; and patients without adverse outcomes. The last group was used as the reference group. The same regression analyses were carried out as described above, but with these four groups of patients as independent predictors (rather than the two groups without specification of timing of occurrence).

$p$ Values $\leqslant 0.05$ were considered to be statistically significant. Both the estimates from the regression analyses and 95\% CIs have been reported.

\section{RESULTS}

The response was high and full information was obtained from 1876 of the $2145(87.5 \%)$ patients during the interviews. Postdischarge adverse outcomes were reported by 465 (24.8\%) respondents. One or more inhospital adverse outcomes occurred in $294(15.7 \%)$ respondents, of whom 101 (5.4\%) also reported post-discharge adverse outcomes (table 1). The most common adverse outcomes in hospital and after discharge were similar, with infections and dysfunction forming about half the adverse outcomes. Post-discharge adverse outcomes more often consisted of wound healing problems ( $8.1 \%$ vs $4.6 \%$ during hospitalisation) and of symptoms without a diagnosis-for example, fever or pain ( $17.4 \%$ vs $4.6 \%$ during hospitalisation).

Patients with adverse outcomes were older than patients without adverse outcomes (55.1 vs 50.8, respectively, $\mathrm{t}=-5.24$, $\mathrm{p}<0.001$; table 1) and there were relatively more high-risk patients in the adverse outcomes group $(22.5 \%$ vs $10.8 \%$, respectively, $\left.\chi^{2}=45.7, \mathrm{p}<0.001\right)$. The average overall grade of patient evaluation was rather high (7.9) with only $5 \%$ of the patients dissatisfied; however, $41 \%$ of the patients thought that improvements were needed in surgical or nursing care. Patients with adverse outcomes seemed to give slightly lower grades in their overall evaluation, and were more often dissatisfied and more often gave suggestions for improvement. However, given that patients with adverse outcomes were also older, and older 
Table 1 Characteristics of patients with and without adverse outcomes, overall patient evaluation, dissatisfaction and suggestions for improvement (Leiden University Medical Center, 1876 surgical patient admissions during 2003)

\begin{tabular}{|c|c|c|c|c|c|}
\hline & \multirow[b]{2}{*}{$\begin{array}{l}\text { Patients without } \\
\text { adverse outcomes }\end{array}$} & \multicolumn{3}{|c|}{ Patients with adverse outcomes } & \multirow[b]{2}{*}{ Total } \\
\hline & & Only in hospital & $\begin{array}{l}\text { Only after } \\
\text { discharge }\end{array}$ & $\begin{array}{l}\text { In hospital and } \\
\text { after discharge }\end{array}$ & \\
\hline Number & 1218 & 193 & 364 & 101 & 1876 \\
\hline Age at admission (years), mean (SD) & $50.8(17.0)$ & $58.3(16.7)$ & $53.0(17.2)$ & $56.9(15.7)$ & $52.3(17.1)$ \\
\hline Men $(\%)$ & 54.8 & 51.8 & 51.9 & 57.4 & 54.1 \\
\hline ASA class III-V (\%)* & 10.8 & 31.6 & 15.1 & 31.7 & 14.9 \\
\hline Overall grade of patient evaluation, mean (SD) & $8.0(1.3)$ & $8.1(1.3)$ & $7.8(1.4)$ & $7.7(1.6)$ & $7.9(1.3)$ \\
\hline Dissatisfied (\%) & 4.0 & 6.2 & 7.4 & 6.9 & 5.1 \\
\hline Indicating need for improvement in surgical/nursing care (\%) & 39.7 & 39.9 & 45.3 & 47.5 & 41.3 \\
\hline
\end{tabular}

patients tend to give better grades, this difference may be greater. A similar (or opposite) effect may be true for the high-risk patients.

\section{Overall effect of adverse outcomes on patient satisfaction}

To unravel the independent effects of the several patient characteristics and adverse outcomes we carried out multivariable regression analyses in which adverse outcomes were included regardless of the time of occurrence (table 2). Patients with adverse outcomes on average gave $15 \%$ lower grades than patients without adverse outcomes, were twice as likely to be dissatisfied and more often indicated that medical care could be improved (table 2). In addition, older patients and patients with ASA class 1-2 or missing class gave higher (ie, better) grades, and operated patients (in every ASA group) were less often dissatisfied than patients who did not have an operation. To identify why patients with adverse outcomes more often indicated that improvements in care were needed, we explored differences between patients with and without adverse outcomes for the improvement categories described before. Patients with adverse outcomes more often indicated that the patient-caregiver contact (odds ratio (OR) 1.67, 95\% CI 1.19 to 2.34) and medical care (OR 1.95, 1.43 to 2.65) could be improved, in particular, the timing of discharge, which they perceived as being too early (OR 2.26, 1.22 to 4.16 ). They less often suggested management issues as most in need for improvement (OR 0.71, 0.55 to 0.92 ).

\section{Effects of inhospital and post-discharge adverse outcomes on patient satisfaction}

The occurrence of inhospital adverse outcomes alone did not have a significant effect on the overall patient evaluation, dissatisfaction or suggestions for improvement (table 3).
Patients who experienced adverse outcomes only after discharge gave $19 \%$ lower grades and were twice as likely to be dissatisfied compared with patients without any adverse outcomes (table 3 ). Patients with both post-discharge and inhospital adverse outcomes on average gave 33\% lower grades than patients without any adverse outcomes (table 3). The effect on dissatisfaction was not significant although the estimated odds ratio seemed similar to the effect of postdischarge adverse outcomes alone. The large confidence interval suggests that there was more variation among the answers given by this group of patients than among those of patients with post-discharge adverse outcomes only.

Similar findings were obtained when only serious adverse outcomes during admission were considered-that is, adverse outcomes requiring reoperation or resulting in permanent damage or disability: the effect of inhospital adverse outcomes alone on overall evaluation was still not significant $(B=-0.10$, -0.42 to 0.22 ) and neither was the effect on dissatisfaction (OR $1.56,0.53$ to 4.57 ) or on suggestions for improvement (OR 1.00, 0.61 to 1.65$)$. Thus the severity of the inhospital adverse outcome does not explain why no significant effect was found. Furthermore, using a cut-off point of 5 to indicate dissatisfaction did not change the results: only patients with postdischarge adverse outcomes only were significantly more likely to be dissatisfied than patients without any adverse outcomes (OR 2.10, 1.28 to 3.45 ).

In trying to identify which areas of improvement were mentioned by patients with adverse outcomes, we further explored differences between the four groups of patients for the improvement categories that were described before (fig 1). Figure 1 shows that patients with inhospital adverse outcomes alone more often mentioned that patient-caregiver contact could be improved, even though they seemed more satisfied with the information received. Patients with post-discharge

Table 2 Impact of adverse outcomes, regardless of time of occurrence, on overall patient evaluation, dissatisfaction and need for improvement in care (Leiden University Medical Center, 1876 surgical patient admissions during 2003)

\begin{tabular}{|c|c|c|c|}
\hline & Overall patient evaluation & Dissatisfaction & Improvement needed \\
\hline & B $(95 \%$ Cl) & Odds ratio $(95 \% \mathrm{Cl})$ & Odds ratio $(95 \% \mathrm{Cl})$ \\
\hline Adverse outcomes (yes/no) & $-0.149(-0.276 \text { to }-0.022)^{*}$ & $2.01(1.31 \text { to } 3.10)^{*}$ & $1.22(1.00 \text { to } 1.48)^{*}$ \\
\hline Age (years) & $0.005(0.002 \text { to } 0.009)^{*}$ & 1.00 (0.99 to 1.01$)$ & 1.00 (0.99 to 1.00$)$ \\
\hline Gender (women/men) & $-0.038(-0.158$ to 0.082$)$ & 1.45 (0.95 to 2.21$)$ & 1.18 (0.98 to 1.42$)$ \\
\hline ASA class $1-2 \dagger$ & $0.275(0.128 \text { to } 0.421)^{*}$ & $0.37(0.23 \text { to } 0.59)^{*}$ & $1.17(0.94$ to 1.47$)$ \\
\hline ASA class 3-5† & $0.170(-0.028$ to 0.368$)$ & $0.50(0.27 \text { to } 0.94)^{*}$ & 0.94 (0.69 to 1.29$)$ \\
\hline ASA class missing $\dagger$ & $0.503(0.188 \text { to } 0.817)^{*}$ & $0.12(0.02 \text { to } 0.89)^{*}$ & $0.93(0.57$ to 1.52$)$ \\
\hline Model fit & Adjusted $\mathrm{R}^{2}$ & Nagelkerke $R^{2}=0.048$ & Nagelkerke $R^{2}=0.01$ \\
\hline
\end{tabular}

ASA, American Society of Anaesthesiologists.

${ }^{*}$ Differences significant in the regression analyses $(p \leqslant 0.05)$.

†Reference category: patients without operation 
Table 3 Impact of adverse outcome by time of occurrence on overall patient evaluation, dissatisfaction and need for improvement in care (Leiden University Medical Center, 1876 surgical patient admissions during 2003)

\begin{tabular}{|c|c|c|c|}
\hline & Overall patient evaluation & Dissatisfaction & Improvement needed \\
\hline & B $(95 \% \mathrm{Cl})$ & Odds ratio $(95 \% \mathrm{Cl})$ & Odds ratio $(95 \% \mathrm{Cl})$ \\
\hline Inhospital adverse outcomes only $\dagger$ & $0.039(-0.166$ to 0.243$)$ & 1.88 (0.95 to 3.72$)$ & $1.04(0.76$ to 1.43$)$ \\
\hline Adverse outcomes after discharge only $\dagger$ & $-0.193(-0.347 \text { to }-0.039)^{*}$ & $2.02(1.24 \text { to } 3.31)^{*}$ & $1.26(1.00$ to 1.60$)$ \\
\hline Inhospital and after discharge adverse outcomes $†$ & $-0.334(-0.604 \text { to }-0.064)^{*}$ & $2.22(0.95$ to 5.18$)$ & $1.43(0.94$ to 2.16$)$ \\
\hline Age (years) & $0.005(0.002 \text { to } 0.009)^{*}$ & 1.00 (0.99 to 1.01$)$ & 1.00 (0.99 to 1.00$)$ \\
\hline Gender (women/men) & $-0.04(-0.161$ to 0.08$)$ & 1.45 (0.95 to 2.21$)$ & 1.18 (0.98 to 1.43$)$ \\
\hline ASA class I-II & $0.271(0.124 \text { to } 0.417)^{*}$ & $0.37(0.23 \text { to } 0.59)^{*}$ & 1.18 (0.94 to 1.48$)$ \\
\hline ASA class III-V $¥$ & $0.157(-0.043$ to 0.357$)$ & $0.50(0.26 \text { to } 0.95)^{*}$ & $0.95(0.70$ to 1.30$)$ \\
\hline ASA class missing $\ddagger$ & $0.495(0.181 \text { to } 0.81)^{*}$ & $0.12(0.02 \text { to } 0.89)^{*}$ & 0.93 (0.57 to 1.53$)$ \\
\hline Model fit & Adjusted $R^{2}=0.013$ & Nagelkerke $R^{2}=0.048$ & Nagelkerke $\mathrm{R}^{2}=0.011$ \\
\hline
\end{tabular}

ASA, American Society of Anaesthesiologists.

*Differences significant in the regression analyses $(p \leqslant 0.05)$.

†Reference category: patients without any adverse outcomes.

†Reference category: patients without operation.

adverse outcomes alone, on the other hand, more often thought that medical care could be improved and less often mentioned management issues as the aspect most in need of improvement. In particular, these patients more often felt discharged too early (OR 3.26, 1.72 to 6.20).

Finally, patients who experienced both inhospital and postdischarge adverse outcomes more often mentioned that both patient-caregiver contact and medical care could be improved. The effect of time of discharge being too early was not significant (OR 2.36, 0.78 to 7.15 ), with the large confidence interval indicating that the views of this group of patients about the aspects mentioned varied more than those of patients with post-discharge adverse outcomes only.

The question then is whether the patients who experienced adverse outcomes only after discharge had a shorter length of stay or that discharge was merely perceived to be too early (relative to their expectation). For the group of patients without inhospital adverse outcomes, a longer length of stay ( 6 days or more) increased the probability of post-discharge adverse outcomes rather than a shorter length of stay (OR 1.33, 1.04 to 1.70; adjusted for age, gender, ASA class of first operation). Thus it seems that patients perceive discharge being too soon if they later experience adverse outcomes, even if the length of stay was relatively long (rather than shorter).

\section{DISCUSSION}

The present study has shown that adverse outcomes influence patient satisfaction, in particular if these occur after discharge in patients without inhospital adverse outcomes. Patients with postdischarge adverse outcomes only rated the quality of care as lower and were more often dissatisfied. They also indicated more often that medical care could be improved, and in particular felt they had been discharged too early, even though this perception was not supported by the data on actual length of stay. The effect of inhospital adverse outcomes alone on overall evaluation and dissatisfaction was not statistically significant, but these patients more often indicated that the patient-caregiver contact could be improved, even though they were more satisfied with the information they received than the patients without adverse outcomes. Patients with both inhospital and post-discharge adverse outcomes also rated the quality of care as lower, and more often indicated that both the patient-caregiver contact and medical care could be improved.

Post-discharge telephone follow-up after hospitalisation is associated with increased patient satisfaction. ${ }^{13}{ }^{14}$ Therefore, the ratings obtained in the present study may be positively biased and respondents may tend to report fewer negative issues than actually experienced. Since we used the same methods for patients with and without adverse outcomes, it seems improbable this may selectively influence subgroups of patients and thus explain the different evaluations of quality of care. If the reporting of post-discharge adverse outcomes had triggered patients who experienced them not to hold back the negative issues, and made them feel justified to give a lower grade, this may have explained our findings. A different ordering of the questions in the interview-that is, questions on patient satisfaction before those on adverse outcomes-may have given different results.

Jenkinson and colleagues' results suggested that patient satisfaction scores present a limited and optimistic picture, ${ }^{1}$ and that detailed questions about specific aspects of patients' experiences are more likely to highlight areas for improvement
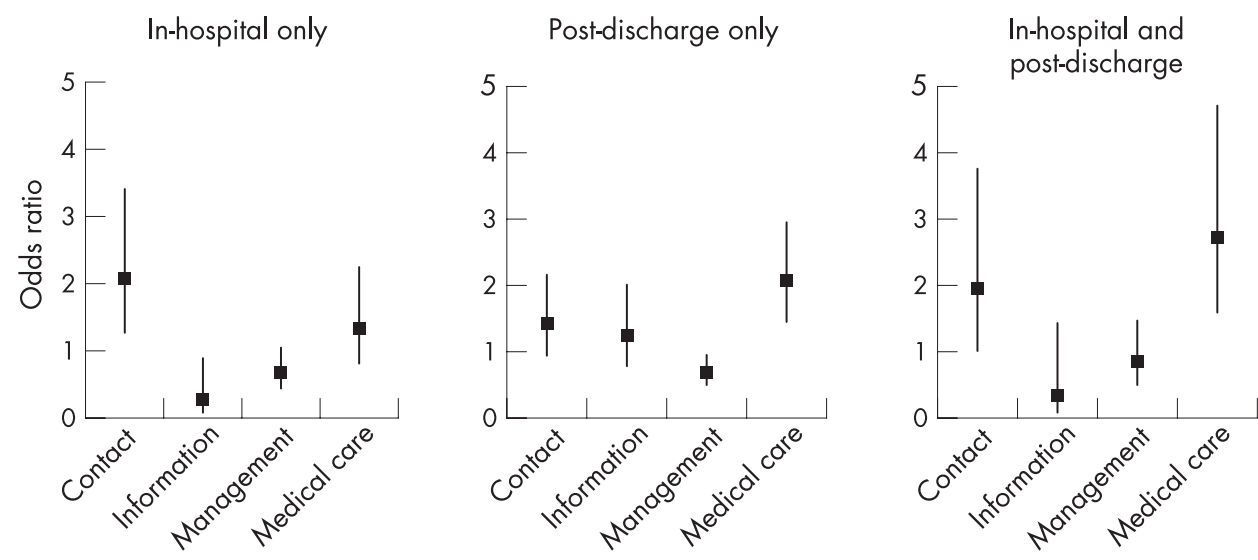

Figure 1 Differences between patients with and without adverse outcomes, occurring in hospital and/or after discharge, in the improvement categories (ORs adjusted for age, gender and ASA class of first operation, with $95 \% \mathrm{Cls}$ ). 


\section{Key messages}

- Patient satisfaction is an important indicator of quality of care. To patients, adverse outcomes are relevant as they may increase the burden of disease. Sicker patients have a lower appreciation of the quality of healthcare.

- The present study shows that adverse outcomes diminish patients' appreciation of the quality of care, in particular, those occurring after discharge. Patients with postdischarge adverse outcomes alone more often indicated that medical care could be improved, and felt that they were discharged too early even though this perception was not supported by data on actual length of stay.

- The effect of inhospital adverse outcomes on patient satisfaction was not statistically significant, irrespective of level of severity, but these patients more often indicated that patient-caregiver contact could be improved even though they were more satisfied with the information provided.

- These results suggest that we need to inform and prepare patients at discharge that adverse outcomes do occasionally occur after discharge, but that this does not require or justify longer hospitalisation.

- We recommend that caregivers make sure at discharge that patients have the information they need to avoid or help to deal with post-discharge adverse outcomes if these occur.

in the quality of care. In the present study only $5 \%$ of patients were dissatisfied, but over $40 \%$ mentioned that some aspect of care could be improved. Patients are reluctant to express criticisms, but if they are supported, reassured and given the opportunity to provide a rationale for their evaluations of their care, it is likely to facilitate such expression. ${ }^{15}$ We think that the positive framing of our questions, aimed at potential improvement of care for future patients, may have created such an opportunity and therefore provided us with more information than the overall evaluation scores. However, given our results with respect to the improvements suggested by patients, more information on the actual communication and specific information provided at discharge would have been helpful to explain our findings-for example, when information at discharge was given but perhaps not understood by the patient. Since such data were not collected, we can only make general rather than specific recommendations on things that need to change around the time of discharge. Future research on the effect of adverse outcomes on patient satisfaction should include more of these specific questions, as mentioned by Jenkinson, besides the overall patient evaluation.

We found that occurrence of post-discharge adverse outcomes alone had a negative influence on the overall evaluation of quality of care and increased the probability of dissatisfaction. The effect of inhospital adverse outcomes alone was not statistically significant. One explanation for these findings could be that inhospital and post-discharge adverse outcomes were reported by doctors and patients, respectively. Therefore, patients may have been unaware of some of the inhospital adverse outcomes, in which case no influence on subjective overall evaluation is expected. On the other hand, all postdischarge adverse outcomes were reported (and thus experienced) by patients themselves, and these may have negatively influenced patient satisfaction. Also, post-discharge adverse outcomes may be remembered better, as they would have occurred more recently. This explanation is unlikely for two reasons. First, the definition of an adverse outcome was explained to the patient during admission and again at the start of the interview after discharge. Second, and more importantly, we found similar results if only more serious adverse outcomes were considered, such as those that required additional surgery, which did not go unnoticed, and were almost certainly not forgotten by the time of the interview.

We therefore conclude that post-discharge adverse outcomes diminish patients appreciation of the quality of care. In addition, these patients more often indicated that medical care should be improved, in particular, not discharging patients too early. So it seems that patients expect to be discharged only after all the risks have been dealt with, and that any postdischarge adverse outcomes needing treatment are seen as reduced quality of care. This suggests that we need to inform and prepare patients at discharge that adverse outcomes do occasionally occur after discharge, but that this does not require or justify longer hospitalisation but can be followed up at the outpatient clinic. Future studies should include the specific information that patients receive at discharge. We also recommend that caregivers make sure when discharging patients, that patients have (understood) all the information they need to prevent post-discharge adverse outcomes from occurring, or help to deal with them if they do occur.

\section{ACKNOWLEDGEMENTS}

We thank Dr W Otten and Dr C E Jacobi for their comments on an earlier draft of this paper, and Professor $\mathrm{H}$ van Houwelingen for statistical advice.

\section{Authors' affiliations}

Perla J Marang-van de Mheen, Nanny van Duijn-Bakker, Department of Medical Decision Making, Leiden University Medical Center, Leiden, The Netherlands

Job Kievit, Department of Surgery, Leiden University Medical Center, Leiden, The Netherlands

Funding: Leiden University Medical Centre, division I.

Competing interests: None.

\section{REFERENCES}

1 Jenkinson C, Coulter A, Bruster S, et al. Patients' experiences and satisfaction with health care: results of a questionnaire study of specific aspects of care. Qual Saf Health Care 2002;11:335-9.

2 Delbanco T. Hospital medicine: understanding and drawing on the patient's perspective. Am J Med 2001;111:S2-4.

3 Crow R, Gage H, Hampson S, et al. The measurement of satisfaction with healthcare: implications for practice from a systematic review of the literature. Health Technol Assess 2002;6:1-250.

4 Marang-van de Mheen PJ, van Hanegem N, Kievit J. Effectiveness of routine reporting to identify minor and serious adverse outcomes in surgical patients. Qual Saf Health Care 2005;14:378-82.

5 Marang-van de Mheen PJ, Stadlander MC, Kievit J. Adverse outcomes in surgical patients. Implementation of a nationwide routine reporting system. Qual Saf Health Care 2006; 15:320-4.

6 Brennan TA, Leape LL, Laird NM, et al. Incidence of adverse outcomes and negligence in hospitalized patients: results of the Harvard Medical Practice Study I. N Engl J Med 1991;324:370-6.

7 Gawande AA, Thomas EJ, Zinner MJ, et al. The incidence and nature of surgical adverse outcomes in Colorado and Utah in 1992. Surgery 1999;126:66-75.

8 Kable AK, Gibberd RW, Spigelman AD. Adverse outcomes in surgical patients in Australia. Int J Qual Health Care 2002;14:269-76.

9 Davis P, Ley-Yee R, Briant R, et al. Adverse outcomes in New Zealand Public Hospitals I: occurrence and impact. N Z Med J 2002;1 15:U271-9.

10 Sixma HJ, van Campen C, Kerssens JJ, et al. [The QUOTE-questionnaires. Quality of care from a patients perspective; 4 new instruments] [in Dutch]. Utrecht: Nivel, 1998:1-143.

11 Winter-van der Meer ACM, Kleefstra SM, Kool RB, et al. [Satisfied, but it could be better. Satisfaction of patients in university medical centres] [in Dutch]. Report commissioned by the Netherlands Federation of University Medical Centers. Utrecht: Prismant, 2004:13.

12 American Society of Anesthesiologists. New classification of physical status. Anesthesiology 1963;24:111.

13 Dudas V, Bookwalter T, Kerr KM, et al. The impact of follow-up telephone calls to patients after hospitalization. Am J Med 2001;111:S26-30. 
14 Nelson JR. The importance of postdischarge telephone follow-up for hospitalists: a view from the trenches. Am J Med 2001;111:S43-4.
15 Staniszewska S, Henderson L. Patients evaluations of their health care: the expression of negative evaluation and the role of adaptive strategies. Patient Educ Couns 2004:55:185-92.

\section{APPENDIX A}

\section{CATEGORIES AND ITEMS OF IMPROVEMENT SUGGESTED BY PATIENTS IN SURGICAL CARE AND NURSING CARE}

\begin{tabular}{ll}
\hline Category of improvement & Issues that could be improved \\
\hline $\begin{array}{c}\text { Surgical care } \\
\text { Contact with surgeons }\end{array}$ & $\begin{array}{l}\text { Individual doctor-patient relationship (eg, doctors not friendly, not introducing themselves, } \\
\text { not enough time) } \\
\text { Large number of doctors (eg, large group of doctors at bedside, nobody introduces } \\
\text { themselves) } \\
\text { Personal attention for patient (eg, doctors should listen more to patient, not taking } \\
\text { complaint seriously) } \\
\text { Other personal treatment issues relating to doctors }\end{array}$
\end{tabular}

Transfer of information from surgeon to patient

Between doctor and patient (eg, not enough explanation, talking with each other but not with patient)

Clear information before surgery

Information on (course of) treatment (eg, treating surgeon has not explained outcome of surgery)

Information on (results of) tests (eg, information too limited)

Information at discharge (eg, how wound should be treated at home)

Other information from doctor to patient

Management relating to Information transferral between doctors (eg, disagreement on treatment policy between treatment doctors, telling the same thing over and over again Information transferral to nurses during admission Information transferral at discharge (eg, to GP)

Waiting list of surgery

Waiting time at admission (eg, had to come early and then wait for treatment for a long time)

Waiting long for a doctor on day of discharge

Admitted and send home (eg, due to other (emergency) surgery)

Doctors changing all the time

Other management issues relating to doctors

Medical care by surgeons Timing of discharge, resulting in more medical complaints (eg, discharged too early, resulting in infection)

Performance of medical procedure (eg, procedure caused a lot of pain, anaesthesia went wrong, ugly scar)

Management by doctors resulting in health disadvantage to patient leg, had to pay attention themselves whether the right treatment was received, eg, epidural or general anaesthesia)

Other dissatisfaction with treatment by doctors

Other

Other improvements in surgical care mentioned

Nursing care

Contact with nurses

Nurse-patient relationship (eg, unfriendly nurse)

No time to talk, little time for patient, impersonal

Not taking patient seriously, poor listening

Other personal treatment issues relating to nurses

Transfer of information from a nurse to patient

Between nurse and patient (eg, not enough explanation, wrong information) Information on use of medication

Information on course of illness or discharge

Information on (course of) treatment

Other information given by nurses to patients

Management relating to treatment

Information transferral to doctors during admission, or with other nurses (eg, patient giving the same information several times)

Nurses at central counter or in hall way spending too much time talking

Too little privacy and quiescence (eg, due to visitors or taking care of other patients)

Waiting time (eg, before being treated, being left alone, responding late to bell)

Management relating to discharge

Other management issues relating to nurses

Medical care by nurses Wound treatment (eg, wound treated once instead of twice each day)

Other medical treatment (eg, mistakes in drawing blood or setting up infusion)

General treatment (eg, not being washed properly)

Medication (eg, wrong medication given or at wrong time)

Other dissatisfaction with treatment by nurses 